\title{
LOW MASS WHITE DWARFS IN BINARIES
}

T. R. MARSH ${ }^{1}$, V. S. DHILLON ${ }^{2}$, S. R. DUCK ${ }^{3}$

1. University of Southampton, Dept. of Physics, Highfield, Southampton, SO17 1BJ, UK

2. Royal Greenwich Observatory, Apartado de Correos 321, Santa Cruz de La Palma, 38780 Tenerife, Canary Islands, Spain

3. University of Oxford, Dept. of Physics, Nuclear and Astrophysics Laboratory, Keble Road, Oxford, OX1 3RH, UK

\begin{abstract}
The lowest mass white dwarfs that can have been produced by single star evolution in the lifetime of the Galaxy have masses of about $0.53 \mathrm{M}_{\odot}$. There are however several white dwarfs known with significantly lower masses. Evolution in a binary provides a straightforward explanation as the star can lose its envelope before ever burning helium. The products are expected to be short-period binary stars. To test this we have looked at 15 such stars and have found that 8 of them are close binaries. Thus binary evolution does have a major role in the formation of low-mass white dwarfs. We discuss whether the non-detections could also be binary stars.
\end{abstract}

\section{Introduction}

A consistent prediction of binary star evolution is the existence of white dwarf/white dwarf binary stars with orbital periods of order hours to days. Such systems which are descended from much more widely separated mainsequence star binaries are expected to exist in significant numbers, and of order 10 to $20 \%$ of all white dwarfs may reside in them (Iben 1991). Until recently though, out of over 1000 confirmed white dwarfs only 2 short period, double-degenerate systems were known [L870-2: $P=1.56 \mathrm{~d}$, (Saffer et al. 1988); and WD 0957-666: $P=1.18 \mathrm{~d}$ (Bragaglia et al. 1990)].

The paucity of known short-period, double-degenerate binaries exists in spite of several surveys devoted to finding them (Robinson \& Shafter (1987); Foss et al. (1991); Bragaglia et al. (1990)). However, all of these surveys were designed to detect short-period degenerate pairs with a com- 
bined mass exceeding the Chandrasekar limit as such systems are possible progenitors of the Type Ia supernovae. The combination of short-period and high mass produces high velocities which do not require great sensitivity to detect. However, the same features are rare amongst the expected population of double-degenerates leaving the majority of lower mass, longer period systems undetected.

In an effort to test the theory more stringently, we decided to concentrate upon the low mass tail of the white dwarf distribution (Bergeron, Saffer \& Liebert 1992) since this should be dominated by the products of binary star evolution. Detailed write-ups of some of this work are already appearing in the literature (Marsh 1995) so in this paper we take more of an overview of the results so far.

\section{Observations and their analysis}

We have now had 2 runs of 3 nights on the $4.2 \mathrm{~m} \mathrm{WHT}$ and 2 runs of 1 week each on the $2.5 \mathrm{~m} \mathrm{INT}$ (the second of which has yet to be analysed). On the WHT we are able to cover simultaneously $\mathrm{H} \alpha$ for radial velocity measurement and the $I$-band for late-type features. On the INT we can only cover one or other of these at a time. We use a dispersion of $0.4 \AA \mathrm{pixel}^{-1}$ at $\mathrm{H} \alpha$ with a FWHM of 2 pixels in every case.

This moderately high dispersion is needed to make the most of the narrow core in $\mathrm{H} \alpha$ displayed by many DA (hydrogen lines only) white dwarfs. All of our discoveries have come from the $\mathrm{H} \alpha$ region, even the white dwarf/M dwarf binary (Table 1). An important part of the analysis has been the development of a multi-Gaussian fitting routine which permits varied constraints to be applied.

The combination of the Gaussian fit velocities, the high dispersion and the narrow core of $\mathrm{H} \alpha$ have produced typical radial velocity uncertainties in the range 2 to $5 \mathrm{~km} \mathrm{~s}^{-1}$. These are low values given the huge breadth of the pressure broadened lines in white dwarfs and we believe that much of our success can be put down to them.

\section{Results}

We have now analysed data on 15 white dwarfs. Table 1 summarises our results on these systems. The table shows that out of the 15 systems, 8 are binaries. Of the binaries, 7 are double-degenerates and one is a detached white dwarf/M dwarf binary. The latter system is certainly interesting as it is the shortest period such system known and falls exactly in the centre of the period gap of cataclysmic variable stars, however we will not discuss it further here. The new systems have extended the double-degenerate period range to shorter and longer periods with the range now extending from 
TABLE 1. Low mass white dwarfs studied to date.

\begin{tabular}{lccccl}
\hline $\begin{array}{l}\text { Object } \\
\text { WD }\end{array}$ & $\begin{array}{c}M_{\mathrm{W}} \\
\mathrm{M}_{\odot}\end{array}$ & Prob & Binary & $\begin{array}{c}\text { Period } \\
(\mathrm{d})\end{array}$ & Comments \\
\hline $0136+768$ & 0.44 & 0.82 & Yes & $?$ & Double-lined WD/WD \\
$0147+674$ & 0.45 & 0.72 & No & - & \\
$0316+345$ & 0.40 & 0.94 & No & - & \\
$0339+523$ & 0.34 & 0.89 & No & - & \\
$0453+418$ & 0.43 & 0.94 & No & - & \\
$0710+741$ & 0.35 & 0.90 & Yes & 0.103 & WD $+0.1 \mathrm{M}_{\odot} \mathrm{M}$ dwarf \\
$0839+231$ & 0.48 & 0.89 & No & - & \\
$1101+364$ & 0.31 & 0.84 & Yes & 0.145 & Double-lined WD/WD \\
$1241-010$ & 0.31 & 0.93 & Yes & 3.347 & Single-lined WD/WD \\
$1317+453$ & 0.33 & 0.96 & Yes & 4.872 & Single-lined WD/WD \\
$1353+409$ & 0.40 & 0.87 & No & - & \\
$1614+136$ & 0.33 & 0.90 & No & - & \\
$1713+332$ & 0.35 & 0.95 & Yes & 1.127 & Double-lined WD/WD \\
$2032+188$ & 0.36 & 0.92 & Yes & $?$ & Single-lined WD/WD \\
$2331+290$ & 0.39 & 0.62 & Yes & 0.167 & Single-lined WD/WD \\
\hline
\end{tabular}

$3.47 \mathrm{~h}$ to $4.87 \mathrm{~d}$. This is significant when we compare with the theoretically expected distribution which is limited in extent.

In Fig. 1 we show the 7 systems of known orbital period (including L870-2 and WD 0957-666) along with the expected distribution from Yungelson et al. (1994). The observations and theory are in surprising agreement given the many uncertainties contained in the theoretical prediction. Double white dwarf binaries pass through a stage in which they orbit within a single envelope. The efficiency with which the envelope is ejected has a significant effect upon the final orbital period. If the envelope is ejected inefficiently, the binary shrinks greatly and the orbital period becomes very short. The observed periods suggest a fairly efficient ejection; Yungelson et al. (1994) used $\alpha_{\mathrm{CE}}=1$ for the distribution plotted in Fig. 1.

We have no evidence for companions in $7 / 15$ systems. Table 1 lists our detection probabilities based upon two $0.4 \mathrm{M}_{\odot}$ white dwarfs in randomly inclined orbits following Yungelson et al.'s period distribution. We should have detected more than 8 systems. These stars may yet be binaries however 


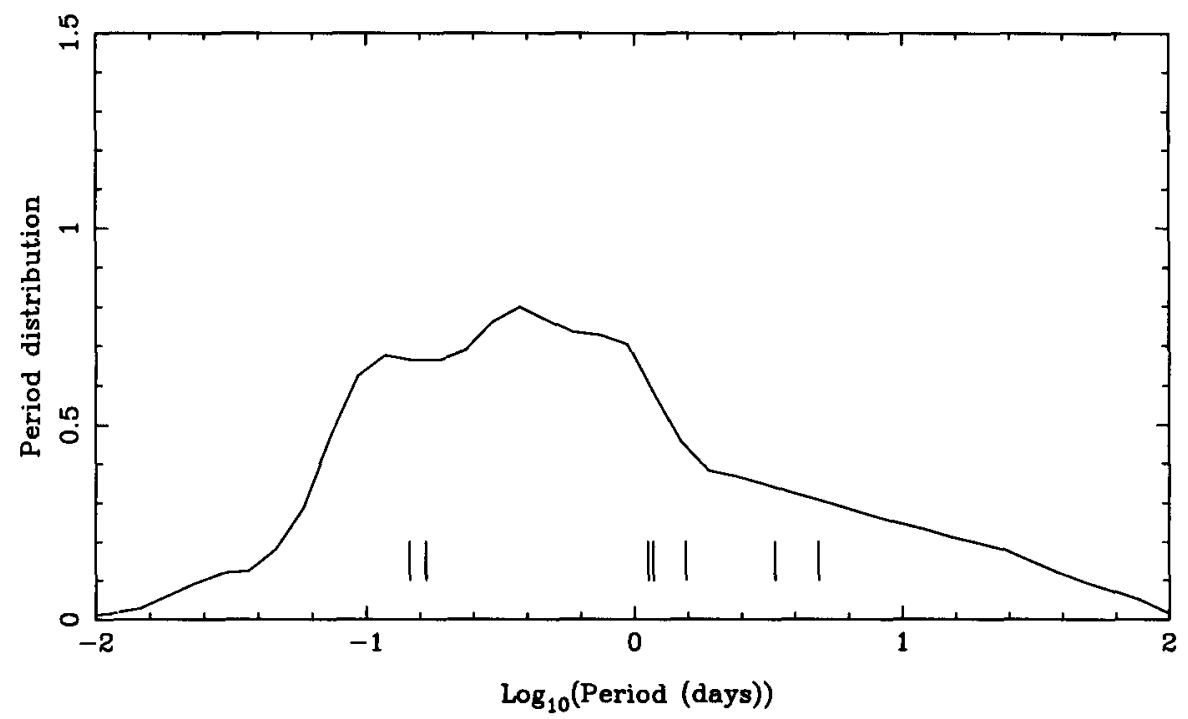

Figure 1. Known orbital periods compared with theoretical prediction.

because symmetric double-lined systems are less likely to be detected and there may be a longer period tail to the distribution than the theoretical distribution suggests. However, there is considerable room for improving the constraints upon these systems and more work is needed on them.

The only other indications of discrepancy between theory and observations are the two measured mass ratios for L870-2 and WD $1101+364$ of $0.90 \pm 0.04$ and $0.87 \pm 0.03$ which are both in the wings of the theoretical prediction (Han, Podsiadlowski \& Eggleton 1995).

\section{Conclusion}

Low mass white dwarfs are predominantly formed in binary stars.

\section{References}

Bragaglia, A., Greggio, L., Renzini, A., D’Odorico, S., 1990, Ap. J., 365, L13

Bergeron, P., Saffer, R.A., Liebert, J., 1992, Ap. J., 394, 228

Foss, D., Wade, R.A., Green, R.F., 1991, Ap. J., 374, 281

Han, Z., Podsiadlowski, P., Eggleton, P.P., 1995, MNRAS, 272, 800

Iben, I., 1991, Ap. J. Supp., 76, 55

Marsh, T.R., 1995, MNRAS, 275, L1

Robinson, E.L., Shafter, A.W., 1987, Ap. J., 322, 296

Saffer, R.A., Liebert, J., Olszewski, E., 1988, Ap. J., 334, 947

Yungelson, L.R., Livio, M., Tutukov, A.V., Saffer, R., 1994, Ap. J., 420, 336 